\title{
Impact of aerobic exercise on muscle mass in patients with major depressive disorder: a randomized controlled trial
}

This article was published in the following Dove Press journal:

Neuropsychiatric Disease and Treatment

\author{
Arno Kerling' \\ Dagmar Hartung ${ }^{2}$ \\ Brendon Stubbs 3,4 \\ Momme Kück' \\ Uwe Tegtbur' \\ Lena Grams' \\ Thomas Sanjay Weber- \\ Spickschen' \\ Kai G Kahl ${ }^{5}$ \\ 'Institute of Sport Medicine, \\ Hannover Medical School, Hannover, \\ Germany; ${ }^{2}$ Institute for Diagnostic \\ and Interventional Radiology, \\ Hannover Medical School, Hannover, \\ Germany; ${ }^{3}$ Physiotherapy Department, \\ South London and Maudsley NHS \\ Foundation Trust, Denmark Hill, \\ London, UK; ${ }^{4} \mathrm{Health}$ Service and \\ Population Research Department, \\ Institute of Psychiatry, King's College \\ London, London, UK; ${ }^{5}$ Department \\ of Psychiatry, Social Psychiatry and \\ Psychotherapy, Hannover Medical \\ School, Hannover, Germany
}

Correspondence: Arno Kerling Institute of Sports Medicine, Hannover Medical School, Carl-Neuberg-Str I, Hannover 30625, Germany Tel +49 5I I 5325499

Email kerling.arno@mh-hannover.de
Objective: Sarcopenia leads to physical function impairment and at least to increasing all-cause mortality. There are notes on reduced muscle mass in patients with major depressive disorder (MDD). Whether an exercise intervention counteracts low muscle mass in patients with MDD has not been studied so far. Therefore, our study aimed at examining effects of regular aerobic exercise training on muscle mass in patients with MDD.

Participants and methods: Thirty inpatients with MDD were included in the study, of which 20 received an additional supervised exercise program. Ten patients obtained treatment as usual. Muscle mass was measured using MRI before and 6 weeks after the training period (3 times per week for 45 minutes).

Results: We found a significant effect of the exercise intervention on the amount of muscle mass depending on age, body mass index, and the physical activity score $(P=0.042)$.

Conclusion: Among other positive effects, regular exercise increases muscle mass in patients with MDD and, therefore, should be recommended as an additional treatment tool.

Keywords: muscle mass, depression, sarcopenia, aerobic exercise

\section{Introduction}

There is an increasing body of evidence that depression is associated with a variety of physical conditions and multimorbidity. ${ }^{1}$ In particular, the prevalence of cardiometabolic diseases is higher in major depressive disorder (MDD), ${ }^{2}$ and this is associated with an elevated risk of premature mortality. ${ }^{3,4}$ Furthermore, increased intra-abdominal and epicardial adipose tissue are key risk factors for cardiometabolic disorders and are increased in patients with MDD. ${ }^{5}$

Considerably less is known about the relationship between depression and skeletal muscle function. However, recent studies have suggested that muscle mass was reduced in elderly Chinese patients of both $\operatorname{sexes}^{6}$ and in middle-aged depressed man. ${ }^{7}$ Depression is also associated with reduced bone mineral density and an increased risk of osteoporotic fracture risk. ${ }^{8,9}$

With increasing age, after a peak in the early adulthood, there is a decrease of $\sim 30 \%$ in muscle strength and a $40 \%$ reduction in muscle mass up to the seventh decade, ${ }^{10}$ with a higher risk for the development of sarcopenia. A recent meta-analysis found evidence of high comorbidity with frailty in older people with depression. ${ }^{11}$ In patients with depression, low cardiorespiratory fitness levels and a sedentary lifestyle have been described. ${ }^{12,13}$ These factors may particularly increase the risk of depletion in the skeletal 
system. In addition, depression ${ }^{14}$ as well as sarcopenia ${ }^{15}$ and frailty ${ }^{16}$ may share common inflammatory pathways.

Depression has been associated with increased levels of cortisol. ${ }^{17}$ Glucocorticoids are described to induce protein degradation in skeletal muscles and, therefore, lasting high cortisol levels may at least, in part, influence muscle mass. ${ }^{18}$

Several studies have demonstrated that exercise interventions influence the cortisol levels. After aerobic exercise was performed at moderate intensity, reduced salivary cortisol levels were found as well as an improvement of depressive symptoms. ${ }^{19}$ Glucocorticoid sensitivity and mRNA levels of the glucocorticoid receptor can be reduced after a standardized prolonged physical training. ${ }^{20}$

Aerobic exercise also has been shown to improve symptoms of depression, cardiorespiratory fitness levels, brainderived neurotrophic factor serum concentrations, and risk factors of the metabolic syndrome and to reduce the amount of epicardial and subcutaneous adipose tissue. ${ }^{12,21-25}$ While there is also robust evidence that exercise can improve muscle function in the general population, there is little interventional research that has considered this in people with MDD.

Given the aforementioned, the aim of this study was to examine if regular aerobic training can improve muscle mass in patients with MDD.

\section{Participants and methods Participants}

The study was authorized by the Ethics Committee of the Hannover Medical School, and all participants gave written informed consent prior to study entry. All patients took part in a randomized pilot trial comparing the effects of adjunctive aerobic exercise on physiological and psychological parameters in depression as described before. In short, the initial study aimed at examining the psychological and physiological effects of a structured, monitored exercise intervention in depressed inpatients. In the original study, 22 patients with MDD received additional exercise treatment (EXERCISE), and 20 MDD patients received treatment as usual (TAU). ${ }^{21}$ As secondary outcomes, the amount of intraabdominal adipose tissue, pericardial adipose tissue, and muscle mass was measured. ${ }^{23}$ Of the original study group, complete data for body composition, including muscle mass, were obtained from 20 patients in the EXERCISE and from 10 in the TAU group. ${ }^{23}$

Study inclusion and exclusion criteria, diagnostic procedures, and recruitment and details concerning exercise treatment are given in detail in Kerling et al's study.$^{21}$ Briefly, inpatients with MDD treated at the Department of Psychiatry, Social Psychiatry and Psychotherapy, Hannover Medical
School were included. Diagnoses were made according to the DSM-IV criteria and confirmed with standardized clinical interviews (SCID I/II; German version). Exclusion criteria were body mass index $\geq 30$, age $<18$ and $>60$ years, current or lifetime cardiovascular or immunological disorders, acute or chronic infectious disease, diabetes mellitus, pregnancy, schizophrenia, mental retardation, bipolar disorder, and current substance abuse or dependency. None of the patients received cardiologic treatments or used $\beta$-blockers. Both groups did not differ in antidepressant pharmacological treatment (85\% in EXERCISE and $90 \%$ in the TAU group). Medication did not change throughout the intervention.

\section{Spiroergometry/EXERCISE intervention}

Detailed information on the exercise program is given in Kerling et al's study. ${ }^{21}$ Briefly, the physician supervised training program was carried out during inpatient treatment at the MHH Institute of Sports Medicine. It consisted of 3 training sessions per week at moderate intensity, each lasting 45 minutes. A constant load test at $50 \%$ of the maximum workload obtained during the initial incremental exercise test was performed to achieve moderate intensity.

This intensity was above the ventilatory anaerobic threshold and below the anaerobic lactate threshold; so, all patients were trained in the aerobic-anaerobic transition zone.

Exercise started with a 25 -minute lasting session on a bicycle ergometer and was continued for another 20 minutes (at personal preference) on a second endurance machine (eg, treadmill, crosstrainer, and rowing). The intervention lasted 6 weeks, and all the patients completed the study. Patients in the TAU group could participate in the daily activity program (walking, ball games, and stretching exercises for 20 minutes) voluntarily.

Behavioral assessment, implementation of the spiroergometry, and the exact performance of the exercise program are described in Kerling et al's study. ${ }^{21}$

Physical activity before starting the intervention was measured with a 6-point Likert scale and ranged from 1 (never) to 6 (very often).

\section{MRI}

Muscle mass were examined using a 1.5 T MRI scanner (Avanto, Siemens Healthcare, Erlangen, Germany) as described before. ${ }^{7}$ Briefly, a T1-weighted 3D Volume Interpolated Breath-hold Examination Dixon sequence of the lower abdomen in an axial orientation was acquired. Following specifications were used: $\mathrm{TR}=7.5 \mathrm{~ms}, \mathrm{TE}=2.4$ and $4.8 \mathrm{~ms}$, flip angle $=10^{\circ}$, matrix $=320 \times 159$, field of view $=400-460 \mathrm{~mm}$, and slice thickness $=5 \mathrm{~mm}$. In-phase, 
out-of phase, fat, and water images were calculated. Muscle mass was quantified from fat images using an axial slice at the level of the lumbar vertebrae (L3) as reported by Psutka et al. ${ }^{50}$ Therefore, a semi-automatic segmentation using MeVisLab 2.8 vc12-64 (MeVis Medical Solutions AG, Bremen, Germany) was used.

\section{Statistical analysis}

The statistical analysis was performed using SPSS version 22.0 (IBM Corp., Armonk, NY, USA) and GraphPad Prism 6 (GraphPad Software Inc., La Jolla, CA, USA). Data were tested for a normal distribution using the KolmogorovSmirnov test. Group differences concerning age, BMI, and lifestyle factors (physical activity, drinking and smoking behavior) were analyzed using 2-tailed $t$-test. To determine the effects of training on muscle mass, repeated measures analysis of variance (ANOVA) with muscle mass as dependent variable, group as independent variable, and age, BMI, and physical activity as potential confounders were used.

Pearson's product-moment correlation coefficient was used to analyze correlations.

Values are presented as mean \pm SD. All $P$-values $<0.05$ were considered to be significant.

\section{Results}

At study entry, there were no significant differences concerning anthropometric data (Table 1). No differences between the groups concerning medication, physical activity, alcohol consumption, smoking, and the Beck-Depressions-Inventory (BDI-2) and the Montgomery-Asberg Depression Rating Scale (MADRS) sum score could be observed. On average, $>90 \%$ (16.7 of 18) of the possible training units were performed. The participation rate for the voluntarily sessions was about $50 \%$.

\section{Comparison of muscle mass}

Repeated measures ANOVA with muscle mass as dependent variable, group as independent variable, and age, BMI, and

Table I Anthropometric data, muscle mass, and physical activity in the EXERCISE $(n=20)$ and the TAU group $(n=10)$ before starting the intervention

\begin{tabular}{llll}
\hline Measure & EXERCISE $(\mathbf{n}=\mathbf{2 0})$ & TAU $(\mathbf{n}=\mathbf{l 0})$ & P-value \\
\hline Age (years) & $44 \pm 8$ & $38 \pm 9$ & $\mathrm{~ns}$ \\
Male $(\mathrm{n})$ & 12 & 6 & $\mathrm{~ns}$ \\
Height $(\mathrm{cm})$ & $174 \pm 9$ & $172 \pm 9$ & $\mathrm{~ns}$ \\
Weight $(\mathrm{kg})$ & $81 \pm 23$ & $79 \pm 12$ & $\mathrm{~ns}$ \\
BMI $\left(\mathrm{kg} / \mathrm{m}^{2}\right)$ & $26.3 \pm 5.0$ & $26.5 \pm 3.3$ & $\mathrm{~ns}$ \\
Muscle mass $\left(\mathrm{cm}^{2}\right)$ & $121 \pm 26$ & $133 \pm 31$ & $\mathrm{~ns}$ \\
Physical activity & $2.7 \pm 1.6$ & $3.1 \pm 1.8$ & $\mathrm{~ns}$ \\
\hline
\end{tabular}

Note: $E X E R C I S E=$ patients with MDD received additional exercise treatment. Abbreviations: BMI, body mass index; TAU, treatment as usual; MDD, major depressive disorder; ns, not significant.
Table 2 Muscle mass in EXERCISE $(n=20)$ and TAU group $(n=10)$ before and after the intervention

\begin{tabular}{|c|c|c|c|c|c|}
\hline \multirow[t]{2}{*}{$\begin{array}{l}\text { Muscle mass } \\
\left(\mathrm{cm}^{2}\right)\end{array}$} & \multicolumn{2}{|c|}{$\begin{array}{l}\text { EXERCISE } \\
(n=20)\end{array}$} & \multicolumn{2}{|l|}{$\begin{array}{l}\text { TAU } \\
(n=10)\end{array}$} & \multirow[t]{2}{*}{$P$-value } \\
\hline & Mean & SD & Mean & SD & \\
\hline & & & & & 0.042 \\
\hline T0 & $12 \mid$ & 26 & 133 & 31 & \\
\hline TI & 124 & 27 & 129 & 35 & \\
\hline
\end{tabular}

Notes: Time $\times$ group effect was analyzed with repeated measures analysis of variance with age, BMI and the physical activity score as covariates. EXERCISE= patients with MDD received additional exercise treatment. A $P$-value $<0.05$ was considered significant.

Abbreviations: TAU, treatment as usual; MDD, major depressive disorder; BMI, body mass index.

physical activity (before the onset of the exercise program) revealed a significant effect of exercise training on muscle mass $(F=4.6 ; d f=1 ; P=0.042)$ (Table 2).

When BDI sum score at study entry was added as additional potential confounder in the model, again a significant effect of exercise on muscle mass was observed $(F=4.6 ; d f=1$; $P=0.042)$. Adding the post-intervention BDI sum scores to the model, a trend toward a significant effect was observed $(F=4.3 ; d f=1 ; P=0.05)$.

Although muscle mass slightly increased in the EXERCISE group and slightly decreased in the TAU group (Figure 1), post-hoc test revealed no significant group differences (data not shown).

\section{Discussion}

To the best of our knowledge, the current randomized trial is the first to demonstrate that an exercise intervention can increase muscle mass in those with depression compared with TAU. Our results remained evident after controlling for age, BMI, and the physical activity score; furthermore, baseline depression scores had no influence on the results.

The reasons why people with depression may have reduced muscle mass are multifactorial. Besides increasing

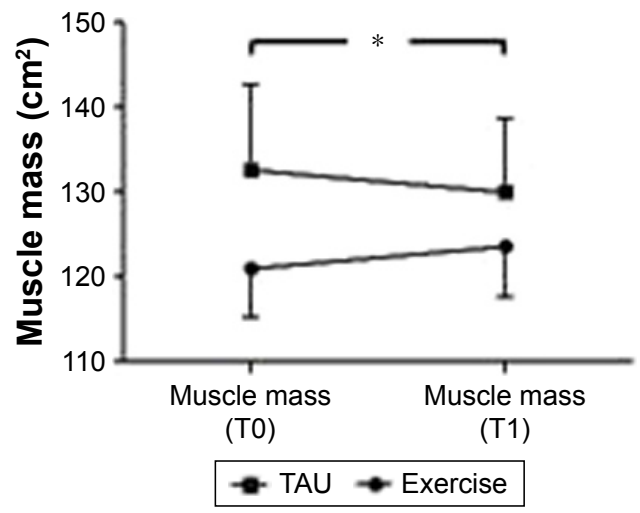

Figure I EXERCISE increases muscle mass in patients with depression. Notes: $E X E R C I S E=$ patients with MDD received additional exercise treatment. $* P=0.042$ Abbreviations: TAU, treatment as usual; MDD, major depressive disorder. 
age, there are many other reasons for decreasing muscle mass and muscle strength like lowered physical activity, multiple physical disorders (pulmonary, cardiac and renal diseases, cancer-associated cachexia, diabetes, Cushing syndrome, sepsis, and burns), or as a physiological response to fasting or malnutrition with low protein and vitamin intake. Most of these pathological conditions are accompanied by an increase in glucocorticoid levels, which results in inhibition of protein synthesis and, together with enhanced proteolysis, leads to muscle atrophy. ${ }^{26}$ Even daily alcohol consumption, cigarette smoking, pro-inflammatory cytokines, and alterations in the endocrine system lead to sarcopenia. ${ }^{26-30}$ Similar changes are seen in patients with MDD, mainly regarding lowered physical capacity, increased rates of substance disorders, higher levels of tumor necrosis factor-alpha (TNF- $\alpha$ ), interleukin-6 (IL-6), and endocrine factors. ${ }^{12,17,31,32}$

Reduced muscle mass is a risk factor for a variety of physical problems, such as osteoporosis, sarcopenia, and frailty. This particularly applies to patients at old age. Fried et $\mathrm{al}^{33}$ defined frailty as a clinical syndrome in which at least 3 of the following 5 criteria are present: unintended weight loss (10 lbs in past year) accompanied by sarcopenia, exhaustion (self-reported), weakness (grip strength), slow walking speed, and low physical activity. The prevalence of depressive symptomatology, frailty, and their simultaneous occurrence is $>10 \%$ in adults $>55$ years of age, and there is a reciprocal interaction between depression and frailty and a robust prospective correlation between depressive symptomatology and increased risk of incident frailty. ${ }^{11,34}$ Another recent meta-analysis suggested that depression is associated with sarcopenia. ${ }^{35}$ Sarcopenia is seen as the biological substrate of physical frailty and is accompanied by loss of functional independence, reduced quality of life, and a higher mortality risk. ${ }^{36-39}$ Thus, our results suggest that it may be possible to reverse some of the reduced muscle mass loss in those with depression with a targeted exercise program.

Aerobic capacity is measured as maximum oxygen consumption $\left(\mathrm{VO}_{2} \max \right)$ and declines after a peak at 25 years from the third decade of life among others due to a reduction of muscle mass and an increase of fat mass. ${ }^{40}$ This decline is $\sim 1 \%$ per year in non-trained persons and in 35\%-50\% due to a decline in fat-free mass. ${ }^{41,42}$ Aerobic capacity is reduced in people who are frail and may be an important factor for frailty in older persons. ${ }^{36}$ Endurance exercise training improves $\mathrm{VO}_{2}$ max, in which the increase seems to be less pronounced in advancing age; ${ }^{43,44}$ therefore, endurance training, especially in patients with depression, should start at an early stage to prevent loss of muscle mass. During weight loss, endurance training can preserve muscle mass contrary to weight loss induced by sole diet. ${ }^{45}$ Regular physical exercise counteracts the catabolic effects of TNF- $\alpha$ and IL- 6 and promotes anabolism through stimulating mechano growth factor and activating satellite cells, which leads to increasing muscle protein synthesis, and muscle mass and strength and, therefore, improves physical frailty. ${ }^{36}$

Additionally, exercise impacts tryptophan and kynurenine metabolism and increases skeletal muscle expression of kynurenine aminotransferases. This leads to a reduction in kynurenine brain accumulation levels (via increased peripheral metabolism), which has been discussed to be the main mechanism in reducing stress-induced depressive symptoms. ${ }^{46,47}$

Although resistance training is the means of choice in preserving or increasing muscle mass, muscle strength, and functional ability, ${ }^{48,49}$ the present endurance training intervention also showed that it could increase muscle mass and, therefore, can be recommended as an effective therapy against sarcopenia in patients with depression.

\section{Limitations}

The main limitation of this study is the small number of patients. In order to improve general applicability of the results, future studies should include more patients using a representative sample best based on data of a multicentric study.

\section{Conclusion}

Endurance training is effective in preserving muscle mass and can be recommended as an effective therapy against sarcopenia in patients with MDD.

\section{Disclosure}

The authors report no conflicts of interest in this work.

\section{References}

1. Stubbs B, Vancampfort D, Veronese N, et al. Depression and physical health multimorbidity: primary data and country-wide meta-analysis of population data from 190593 people across 43 low- and middle-income countries. Psychol Med. 2017;47(12):2107-2117.

2. Vancampfort D, Stubbs B, Mitchell AJ, et al. Risk of metabolic syndrome and its components in people with schizophrenia and related psychotic disorders, bipolar disorder and major depressive disorder: a systematic review and meta-analysis. World Psychiatry. 2015;14(3): 339-347.

3. Walker ER, Mcgee RE, Druss BG. Mortality in mental disorders and global disease burden implications: a systematic review and metaanalysis. JAMA Psychiatry. 2015;72(4):334-341.

4. Correll CU, Solmi M, Veronese N, et al. Prevalence, incidence and mortality from cardiovascular disease in patients with pooled and specific severe mental illness: a large-scale meta-analysis of 3,211,768 patients and 113,383,368 controls. World Psychiatry. 2017;16(2):163-180.

5. Kahl KG, Hueper K, Schweiger U, et al. Pericardial, intra-abdominal, and subcutaneous adipose tissue in patients with major depressive disorder. Acta Psychiatr Scand. 2014;130(2):137-143. 
6. Wu H, Yu B, Meng G, et al. Both muscle mass and muscle strength are inversely associated with depressive symptoms in an elderly Chinese population. Int J Geriatr Psychiatry. 2017;32(7):769-778.

7. Kahl KG, Utanir F, Schweiger U, et al. Reduced muscle mass in middle-aged depressed patients is associated with male gender and chronicity. Prog Neuropsychopharmacol Biol Psychiatry. 2017;76: 58-64.

8. Carlone C, Rusconi AC, Valeriani G, Todini L, Coccanari de' Fornari MA, Biondi M. Osteoporosis and major depression: open debate on a bidirectional relationship. Riv Psichiatr. 2015;50(4):161-167.

9. Stubbs B, Brefka S, Dallmeier D, Stubbs J, VancampfortD, Denkinger MD. Depression and reduced bone mineral density at the hip and lumbar spine: a comparative meta-analysis of studies in adults 60 years and older. Psychosom Med. 2016;78(4):492-500.

10. Rogers MA, Evans WJ. Changes in skeletal muscle with aging: effects of exercise training. Exerc Sport Sci Rev. 1993;21:65-102.

11. Soysal P, Veronese N, Thompson T, et al. Relationship between depression and frailty in older adults: a systematic review and meta-analysis. Ageing Res Rev. 2017;36:78-87.

12. Vancampfort D, Rosenbaum S, Schuch F, et al. Cardiorespiratory fitness in severe mental illness: a systematic review and meta-analysis. Sports Med. 2017;47(2):343-352.

13. Schuch F, Vancampfort D, Firth J, et al. Physical activity and sedentary behavior in people with major depressive disorder: a systematic review and meta-analysis. J Affect Disord. 2017;210:139-150.

14. Köhler CA, Freitas TH, Maes M, et al. Peripheral cytokine and chemokine alterations in depression: a meta-analysis of 82 studies. Acta Psychiatr Scand. 2017;135(5):373-387.

15. Bano G, Trevisan C, Carraro S, et al. Inflammation and sarcopenia: a systematic review and meta-analysis. Maturitas. 2017;96:10-15.

16. Soysal P, Stubbs B, Lucato P, et al. Inflammation and frailty in the elderly: a systematic review and meta-analysis. Ageing Res Rev. 2016;31:1-8.

17. Stetler C, Miller GE. Depression and hypothalamic-pituitary-adrenal activation: a quantitative summary of four decades of research. Psychosom Med. 2011;73(2):114-126.

18. Hu Z, Wang H, Lee IH, Du J, Mitch WE. Endogenous glucocorticoids and impaired insulin signaling are both required to stimulate muscle wasting under pathophysiological conditions in mice. J Clin Invest. 2009; 119(10):3059-3069.

19. Ida M, Ida I, Wada N, Sohmiya M, Tazawa M, Shirakura K. A clinical study of the efficacy of a single session of individual exercise for depressive patients, assessed by the change in saliva free cortisol level. Biopsychosoc Med. 2013;7(1):18-0759-7-18.

20. Sousa E, Silva T, Longui CA, et al. Prolonged physical training decreases mRNA levels of glucocorticoid receptor and inflammatory genes. Horm Res Paediatr. 2010;74(1):6-14.

21. Kerling A, Tegtbur U, Gützlaff E, et al. Effects of adjunctive exercise on physiological and psychological parameters in depression: a randomized pilot trial. J Affect Disord. 2015;177:1-6.

22. Kerling A, Kück M, Tegtbur U, et al. Exercise increases serum brainderived neurotrophic factor in patients with major depressive disorder. $J$ Affect Disord. 2017;215:152-155.

23. Kahl KG, Kerling A, Tegtbur U, et al. Effects of additional exercise training on epicardial, intra-abdominal and subcutaneous adipose tissue in major depressive disorder: a randomized pilot study. J Affect Disord. 2016;192:91-97.

24. Schuch FB, VancampfortD, Richards J, Rosenbaum S, Ward PB, Stubbs B. Exercise as a treatment for depression: a meta-analysis adjusting for publication bias. J Psychiatr Res. 2016;77:42-51.

25. Stubbs B, Rosenbaum S, Vancampfort D, Ward PB, Schuch FB. Exercise improves cardiorespiratory fitness in people with depression: A meta-analysis of randomized control trials. J Affect Disord. 2016; 190:249-253.

26. Fried LP, Tangen CM, Walston J, et al. Frailty in older adults: evidence for a phenotype. J Gerontol A Biol Sci Med Sci. 2001;56(3): M146-M157.

27. Vaughan L, Corbin AL, Goveas JS. Depression and frailty in later life: a systematic review. Clin Interv Aging. 2015;10:1947-1958.
28. Chang KV, Hsu TH, Wu WT, Huang KC, Han DS. Is sarcopenia associated with depression? A systematic review and meta-analysis of observational studies. Age Ageing. 2017;46(5):738-746.

29. Aguirre LE, Villareal DT. Physical exercise as therapy for frailty. Nestle Nutr Inst Workshop Ser. 2015;83:83-92.

30. Landi F, Calvani R, Cesari M, et al. Sarcopenia as the biological substrate of physical frailty. Clin Geriatr Med. 2015;31(3):367-374.

31. Landi F, Calvani R, Tosato M, et al. Impact of physical function impairment and multimorbidity on mortality among community-living older persons with sarcopaenia: results from the ilSIRENTE prospective cohort study. BMJ Open. 2016;6(7):e008281-2015-008281.

32. Sánchez-García S, Gallegos-Carrillo K, Espinel-Bermudez MC, et al. Comparison of quality of life among community-dwelling older adults with the frailty phenotype. Qual Life Res. 2017;26(10):2693-2703.

33. Cohen S, Nathan JA, Goldberg AL. Muscle wasting in disease: molecular mechanisms and promising therapies. Nat Rev Drug Discov. 2015;14(1):58-74

34. Chen L-K, Lee W-J, Peng L-N, et al. Recent advances in sarcopenia research in Asia: 2016 update from the Asian Working Group for sarcopenia. J Am Med Dir Assoc. 2016;17(8):767.e1-7.

35. Steffl M, Bohannon RW, Petr M, Kohlikova E, Holmerova I. Relation between cigarette smoking and sarcopenia: meta-analysis. Physiol Res. 2015;64(3):419-426.

36. Fan J, Kou X, Yang Y, Chen N. microRNA-regulated proinflammatory cytokines in sarcopenia. Mediators Inflamm. 2016;2016:1438686:1-9.

37. Vitale G, Cesari M, Mari D. Aging of the endocrine system and its potential impact on sarcopenia. Eur J Intern Med. 2016;35:10-15.

38. Grant BF, Saha TD, Ruan WJ, et al. Epidemiology of DSM-5 drug use disorder: results from the National Epidemiologic Survey on alcohol and related conditions-III. JAMA Psychiatry. 2016;73(1):39-47.

39. Dowlati Y, Herrmann N, Swardfager W, et al. A meta-analysis of cytokines in major depression. Biol Psychiatry. 2010;67(5):446-457.

40. Lambert CP, Evans WJ. Adaptations to aerobic and resistance exercise in the elderly. Rev Endocr Metab Disord. 2005;6(2):137-143.

41. Proctor DN, Joyner MJ. Skeletal muscle mass and the reduction of VO2max in trained older subjects. J Appl Physiol. 1997;82(5): 1411-1415.

42. Rosen MJ, Sorkin JD, Goldberg AP, Hagberg JM, Katzel LI. Predictors of age-associated decline in maximal aerobic capacity: a comparison of four statistical models. J Appl Physiol. 1998;84(6):2163-2170.

43. Evans EM, Racette SB, Peterson LR, Villareal DT, Greiwe JS, Holloszy JO. Aerobic power and insulin action improve in response to endurance exercise training in healthy 77-87 yr olds. J Appl Physiol. 2005;98(1):40-45.

44. Kohrt WM, Malley MT, Coggan AR, et al. Effects of gender, age, and fitness level on response of VO2max to training in 60-71 yr olds. $J \mathrm{Appl}$ Physiol. 1991;71(5):2004-2011.

45. Weiss EP, Racette SB, Villareal DT, et al. Lower extremity muscle size and strength and aerobic capacity decrease with caloric restriction but not with exercise-induced weight loss. J Appl Physiol. 2007; 102(2):634-640.

46. Agudelo LZ, Femenía T, Orhan F, et al. Skeletal muscle PGC-1 $\alpha 1$ modulates kynurenine metabolism and mediates resilience to stressinduced depression. Cell. 2014;159(1):33-45.

47. Zepf FD, Stewart RM, Guillemin G, Ruas JL. Inflammation, immunology, stress and depression: a role for kynurenine metabolism in physical exercise and skeletal muscle. Acta Neuropsychiatr. 2016; 28(4):244-245.

48. Papa EV, Dong X, Hassan M. Resistance training for activity limitations in older adults with skeletal muscle function deficits: a systematic review. Clin Interv Aging. 2017;12:955-961.

49. Yoshimura Y, Wakabayashi H, Yamada M, Kim H, Harada A, Arai H. Interventions for treating sarcopenia: a systematic review and metaanalysis of randomized controlled studies. J Am Med Dir Assoc. 2017; 18(6):553.e1-16.

50. Psutka SP, Carrasco A, Schmit GD, et al. Sarcopenia in patients with bladder cancer undergoing radical cystectomy: impact on cancerspecific and all-cause mortality. Cancer. 2014;120(18):2910-8. 


\section{Publish your work in this journal}

Neuropsychiatric Disease and Treatment is an international, peerreviewed journal of clinical therapeutics and pharmacology focusing on concise rapid reporting of clinical or pre-clinical studies on a range of neuropsychiatric and neurological disorders. This journal is indexed on PubMed Central, the 'PsycINFO' database and CAS,

and is the official journal of The International Neuropsychiatric Association (INA). The manuscript management system is completely online and includes a very quick and fair peer-review system, which is all easy to use. Visit http://www.dovepress.com/testimonials.php to read real quotes from published authors.

Submit your manuscript here: http://www.dovepress.com/neuropsychiatric-disease-and-treatment-journal 